\title{
Detection of hepatitis B virus pre-S1 and pre-S2 determinants in paraffin wax embedded liver tissue: Importance of reagents used
}

\author{
N Hadzic, A Alberti, B Portmann, D Vergani
}

\begin{abstract}
The presence of pre-S polypeptides in paraffin wax embedded liver sections from the biopsy specimens of 15 hepatitis B surface antigen (HBsAg) seropositive patients (five with chronic persistent hepatitis (CPH), four with chronic active hepatitis (CAH), four with cirrhosis and two "healthy" HBsAg carriers) was investigated using monoclonal antibodies directed to distinct epitopes on pre-S1 (18/7 and TO 606) and pre-S2 (5535 and $Q$ 19/10). Pre-S1 was found in 13 cases when MA 18/7 was used but in only one specimen when TO 606 was used. Pre-S2 was detected in all the biopsy specimens with 5535 and in eight samples with $Q 19 / 10$. Mild enzymatic digestion annulled the staining of all monoclonal antibodies but $Q 19 / 10$. No association was observed between pre-S polypeptide expression and hepatitis $B$ virus (HBV) replication or disease severity.

Pre-S polypeptides can be detected readily in paraffin wax embedded material but the results obtained largely depend on the monoclonal antibodies used.
\end{abstract}

Hepatitis B virion consists of an envelope and a nucleocapsid. The envelope contains the polypeptides $P$ 39/GP 42 (large), GP 33/GP 36 (middle), and P 24/GP 27 (major), encoded, respectively, by pre-S1/pre-S2/S, preS2/S, and S-subregions of the viral genome. ${ }^{1-4}$ The role of pre-S polypeptides in the natural history of the hepatitis B virus (HBV) infection has not yet been fully elucidated, though recent evidence implicates pre-S1, ${ }^{5}$ and less convincingly, pre-S2, ${ }^{6-7}$ in the binding of virions to a putative receptor on the surface of hepatocytes. The distribution of pre-S polypeptides in the liver tissue and its relation to disease severity and HBV replicative activity is a focus of current research. ${ }^{8}$

The intrahepatocytic accumulation of HBV encoded products, especially the surface antigen, ${ }^{9-13}$ has long been related to a "ground glass" appearance of whole or portion of hepatocyte cytoplasm, seen on haematoxylin and eosin stained liver sections, and more clearly shown by either orcein, aldehydefuchsin, or Victoria blue staining.

Using a panel of monoclonal antibodies to well defined pre-S epitopes we investigated the presence and distribution of pre-S polypep- tides in paraffin wax embedded, "ground glass" positive, liver biopsy specimens from patients with a variety of $\mathrm{HBV}$ related liver diseases and diverse HBV replication activity.

The histological material comprised 15 consecutive formalin fixed "ground glass" positive liver biopsy specimens from 15 hepatitis B surface antigen ( $\mathrm{HBsAg}$ ) seropositive patients This selection criterion ensured the presence of cells containing envelope proteins in all the specimens. Eleven patients were men and four women, the age ranging between 17-54 years (mean age $37 \cdot 8$ ). The histological diagnosis, made according to internationally agreed criteria, ${ }^{14}$ was of chronic persistent hepatitis in five, chronic active hepatitis in four, cirrhosis in four and minimal changes ("healthy HBsAg carriers) in two. Seven patients were $\mathrm{HBe}$ antigen positive, while eight were $\mathrm{HBe}$ antibody positive. Eight patients carried hepatitis core antigen ( $\mathrm{HBcAg})$ in their livers as detected by an indirect immunofluorescence technique, ${ }^{15}$ while core antigen was absent from the livers of the remaining seven patients (table 1).

\section{MONOCLONAL ANTIBODIES TO PRE-S} DETERMINANTS

We studied pre-S1 using monoclonal antibodies TO 606 and MA 18/7 and pre-S2 using monoclonal antibodies 5535 and Q 19/ 10. TO 606 and 5535 were donated by Sorin Biomedica, Saluggia, Italy; MA $18 / 7$ and Q $19 / 10$ were a gift of Professor Wolfram Gerlich, Göttingen, Germany. The fine specificity of these monoclonal antibodies has previously been described ${ }^{16}$ and was further confirmed in our laboratory by the use of solid phase enzyme linked immunosorbent assay (ELISA) with synthetic pre-S1 and pre-S2 peptides. MA 18/7 reacted with the 12-47 pre-S1 peptide while TO 606 reacted with the C-terminus (peptide 95-117) of pre-S1. Neither of these monoclonal antibodies recognised pre-S2 determinants. Conversely, $Q$ $19 / 10$ and 5535 did not react with pre-S1 peptides but recognised the 120-145 pre-S2 peptide. Table 2 describes the Ig subclass and specificity of the monoclonal antibodies used, as ascertained in an ELISA, in which pre-S peptides were used in solid phase coated on to wells of a microtitre plate. HBsAg was detected using fluorescein isothiocyanate (FITC) conjugated rabbit anti-HBsAg antiserum (Behring, Marburg, Germany).

IMMUNOHISTOCHEMICAL STAINING

In preliminary experiments we selected a 
Table 1 Clinical data and immunoperoxidase staining in decreasing order of intensity

\begin{tabular}{|c|c|c|c|c|c|c|c|}
\hline Age/sex & Histology & $H B e A g$ & anti-HBe & $\begin{array}{l}\text { Intrahepatic } \\
\text { core }^{\star}\end{array}$ & $A S T$ & $\gamma G T$ & $\begin{array}{l}\text { Immunoperoxidase staining } \\
\text { order of positivity }\end{array}$ \\
\hline $\begin{array}{l}21 / M \\
28 / M \\
17 / \mathrm{F} \\
47 / M \\
51 / M \\
30 / M \\
27 / M \\
48 / M \\
29 / F \\
54 / F \\
41 / F \\
50 / M \\
34 / M \\
36 / M \\
54 / M\end{array}$ & $\begin{array}{l}\text { Minimal changes } \\
\text { Minimal changes } \\
\text { CPH } \\
\text { CAH } \\
\text { Cirrhosis }\end{array}$ & $\begin{array}{l}+ \\
+ \\
- \\
- \\
- \\
+ \\
+ \\
+ \\
+ \\
+ \\
+ \\
+ \\
+ \\
+ \\
-\end{array}$ & $\begin{array}{l}- \\
- \\
+ \\
+ \\
+ \\
- \\
+ \\
+ \\
- \\
+ \\
+ \\
- \\
+ \\
+\end{array}$ & $\begin{array}{l}+++ \\
++ \\
+ \\
+ \\
+ \\
+ \\
+ \\
+ \\
- \\
+ \\
+ \\
+ \\
-\end{array}$ & $\begin{array}{r}45 \\
\text { NT } \\
29 \\
23 \\
34 \\
19 \\
165 \\
37 \\
19 \\
126 \\
\text { NT } \\
129 \\
199 \\
\text { NT } \\
68\end{array}$ & $\begin{array}{r}33 \\
\text { NT } \\
\text { NT } \\
27 \\
37 \\
36 \\
44 \\
40 \\
19 \\
463 \\
\text { NT } \\
240 \\
55 \\
\text { NT } \\
39\end{array}$ & $\begin{array}{l}2^{5}>S>2^{\mathrm{Q}}>1^{\mathrm{M}}=1^{\mathrm{T}} \\
2^{5}>S>1^{\mathrm{M}}>2^{\mathrm{Q}}=1^{\mathrm{T}} \\
1^{\mathrm{M}}>S=2^{\mathrm{S}}>1^{\mathrm{T}}=2^{\mathrm{Q}} \\
\mathrm{S}>2^{\mathrm{S}}>2^{\mathrm{Q}}>1^{\mathrm{M}}>1^{\mathrm{T}} \\
S>2^{5}>1^{\mathrm{M}}>2^{\mathrm{Q}}=1^{\mathrm{T}} \\
S>2^{5}>1^{\mathrm{M}}>1^{\mathrm{T}}=2^{\mathrm{Q}} \\
S^{5}>2^{5}>2^{\mathrm{Q}}>1^{\mathrm{M}}>1^{\mathrm{T}} \\
2^{5}>S>1^{\mathrm{M}}>1^{\mathrm{T}}=2^{\mathrm{Q}} \\
S>2^{5}>1^{\mathrm{M}}>2^{\mathrm{Q}}=1^{\mathrm{T}} \\
2^{5}>S=1^{\mathrm{M}}>2^{\mathrm{Q}}>1^{\mathrm{T}} \\
S>2^{5}>2^{\mathrm{Q}}>1^{\mathrm{M}}>1^{\mathrm{T}} \\
S>2^{5}>1^{\mathrm{M}}>2^{\mathrm{Q}}>1^{\mathrm{T}} \\
1^{\mathrm{M}}>2^{5}=2^{\mathrm{Q}}>1^{\mathrm{T}} \\
S>2^{5}>2^{\mathrm{Q}}>1^{\mathrm{T}}>1^{\mathrm{M}} \\
S>1^{\mathrm{M}}>2^{5}>1^{\mathrm{T}}=2^{\mathrm{Q}}\end{array}$ \\
\hline
\end{tabular}

Normal values: aspartate aminotransferase $($ AST) $=10-50 \mathrm{IU} / 1 ; \gamma$-Glutamyltranspeptidase $(\gamma \mathrm{GT})=>55(\mathrm{~F}),>65(\mathrm{M}) \mathrm{IU} / \mathrm{l}$.

Abbreviations: $\mathrm{S}=$ anti-HBs, $1^{\mathrm{M}}=$ anti-pre-S1 $M A 18 / 7,1^{\mathrm{T}}=$ anti-pre-S1 TO 606, $2^{5}=$ anti-pre-S2 5535, $2^{\mathrm{Q}}=$ anti-pre-S2 $Q 19 / 10, \mathrm{NT}=$ not tested Abbreviations: $S=$ anti-HBs, $1^{M}=$ anti-pre-S
^Detected by indirect immunofluorescence.

Table 2 Characterisation of monoclonal antibodies used for detection of pre-S1 and pre-S2 in liver biopsy specimens

\begin{tabular}{lll}
\hline Antibody & Ig isotype & ELISA S:N ratio \\
\hline pre-S1 & & \\
MA 18/7 & IgG1 & $21 \cdot 6$ \\
TO 606 & IgG1 & $11 \cdot 2$ \\
pre-S2 & & \\
Q 19/10 & IgG2 & $33 \cdot 4$ \\
5535 & IgG1 & 42 \\
\hline
\end{tabular}

* Signal to noise ratio (S:N) calculated using identical antibody and antigen (peptide) concentrations and experimental conditions.

wedge liver biopsy specimen from an $\mathrm{HBsAg}$ positive patient containing all $S$ - and pre-S determinants identified by our panel of antibodies. This specimen was used as the positive control substrate throughout the study and to establish the working dilutions of the antibodies. The dilutions of the antibodies giving optimal intensity of immunofluorescence and immunoperoxidase staining were 1 in 20 for TO 606 and 5535, 1 in 200 for MA $18 / 7$ and $Q 19 / 10$, and 1 in 5 for FITC anti-HBsAg. As negative control an HBV irrelevant monoclonal antibody was used in the first step of the immunofluorescence and immunoperoxidase staining on the positive control substrate.

After dewaxing in xylene and passage through graded alcohol solutions the sections were rehydrated in phosphate buffered saline (PBS) $(0 \cdot 15 \mathrm{~mol} / 1, \mathrm{pH} 7 \cdot 2)$. The sections were initially stained in immunofluorescence, using monoclonal antibodies in the first step and FITC conjugated rabbit anti-mouse immunoglobulin antiserum (Dako, Denmark), diluted 1 in 20 , in the second step. Because three sections were mounted on each slide, the staining produced by the reference anti-

Table 3 Intensity of immunoperoxidase staining in 15 liver biopsy specimens treated with anti-pre-S1 and anti-pre-S2 monoclonal antibodies of different epitopic specificity and with polyclonal anti-HBs

\begin{tabular}{llrrr}
\hline Staining intensity & +++ & ++ & + & 0 \\
\hline Anti-pre-S1 (C-terminus) TO 606 & 0 & 0 & 1 & 14 \\
Anti-pre-S1 (N-terminus) $M A$ 18/7 & 2 & 7 & 5 & 2 \\
Anti-pre-S2 5535 & 4 & 10 & 1 & 0 \\
Anti-pre-S2 Q 19/10 & 0 & 3 & 5 & 7 \\
Anti-HBs & 5 & 9 & 1 & 0 \\
\hline
\end{tabular}

HBsAg antiserum could be directly compared with that given by pre-S monoclonal antibodies. After examining the preparations under ultraviolet microscopy (Polyvar, Reichert-Jung, Austria), the sections were stained with peroxidase conjugated swine anti-rabbit antiserum (Dako, Denmark), diluted 1 in 10 in PBS, for 30 minutes at room temperature. After washing in PBS for 30 minutes peroxidase activity was shown by a 10 minute incubation in a fresh solution $(0.6 \mathrm{mg} /$ $\mathrm{ml}$ ) of 3,3'-diamino-benzidine tetrahydrochloride (Sigma, St Louis, Missouri, USA) in PBS, with $0.6 \mu \mathrm{l} / \mathrm{ml}$ of $30 \% \mathrm{H}_{2} \mathrm{O}_{2}$.

The results of both immunofluorescence and immunoperoxidase staining were recorded on a " 0 ", " + ", to " +++ " scale, according to staining intensity. Additional staining experiments were performed on pronase (Sigma) digested sections $(0.05 \%$ pronase for 60 minutes at $37^{\circ} \mathrm{C}$ ). The peroxidase staining pattern was compared with that produced by conventional histological methods (haematoxylin and eosin and orcein).

\section{Results}

The results obtained by immunofluorescence and immunoperoxidase staining were similar, immunoperoxidase showing a consistently better histological definition. We therefore concentrated on the results obtained by this method, which are summarised in tables 1 and 3. Positive staining was observed with all the monoclonal antibodies, although there was considerable variation in both intensity of staining and number of stained hepatocytes according to the monoclonal antibody used. The most intense staining was obtained with the anti-pre-S2 5535 and with the polyclonal anti-HBsAg. The anti-pre-S1 MA 18/7 also stained most sections (only two negative samples), but the intensity of staining was weaker. The second anti-pre-S2 antibody ( $Q$ $19 / 10)$, was positive on eight and negative on seven specimens. The lowest degree of positivity was obtained with the second antipre-S1 antibody TO 606, which stained only one of the 15 samples.

The following order of staining intensity was obtained: anti-HBs > anti-pre-S2 (5535) > anti-pre-S1 (MA 18/7) > anti-pre-S2 (Q 19/ 

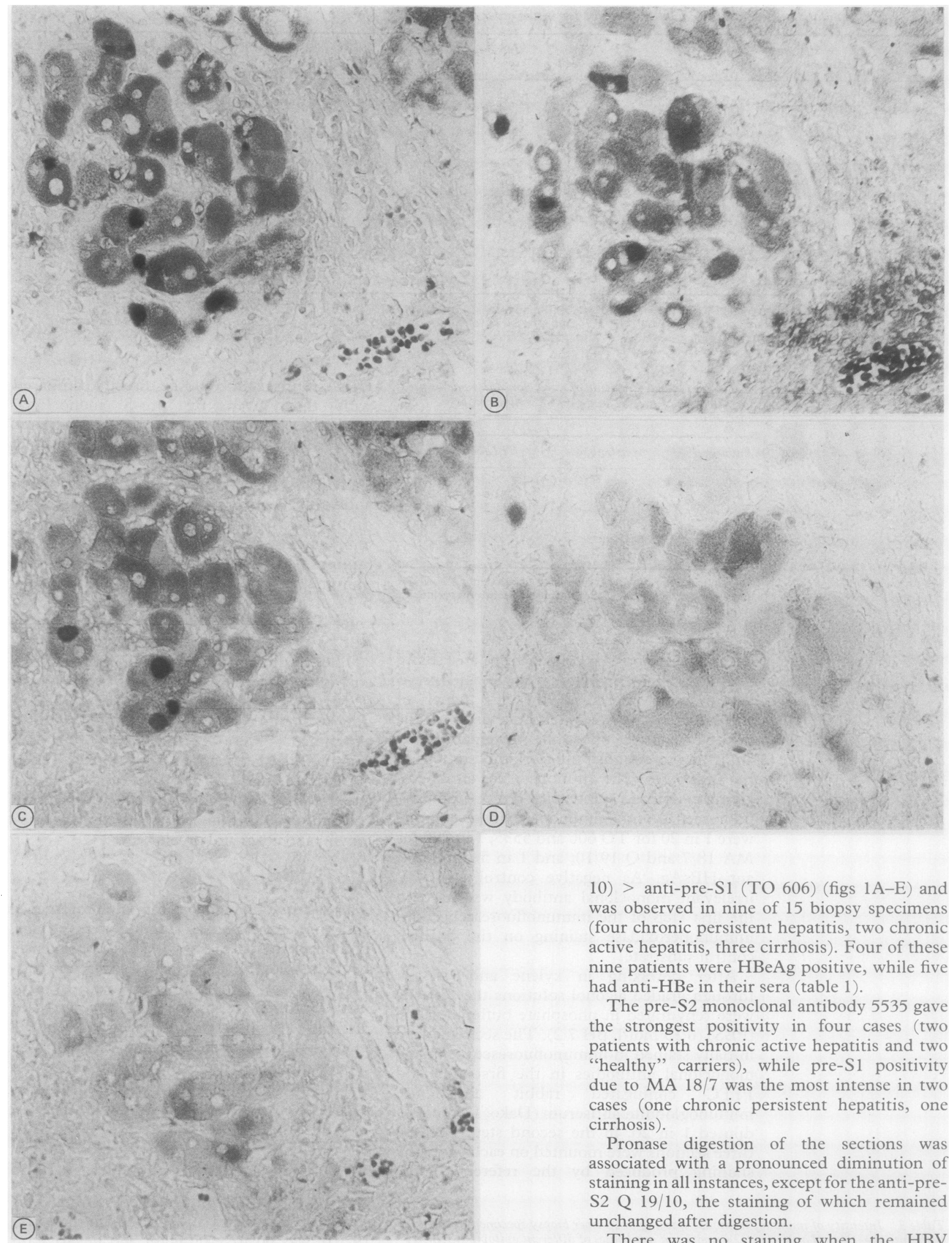

10) > anti-pre-S1 (TO 606) (figs 1A-E) and was observed in nine of 15 biopsy specimens (four chronic persistent hepatitis, two chronic active hepatitis, three cirrhosis). Four of these nine patients were $\mathrm{HBeAg}$ positive, while five had anti-HBe in their sera (table 1).

The pre-S2 monoclonal antibody 5535 gave the strongest positivity in four cases (two patients with chronic active hepatitis and two "healthy" carriers), while pre-S1 positivity due to MA $18 / 7$ was the most intense in two cases (one chronic persistent hepatitis, one cirrhosis).

Pronase digestion of the sections was associated with a pronounced diminution of staining in all instances, except for the anti-preS2 Q 19/10, the staining of which remained unchanged after digestion.

There was no staining when the HBV irrelevant monoclonal antibody was used in the first step of either the immunofluorescence or the immunoperoxidase technique.

The pattern of distribution of both immunofluorescence and peroxidase staining was similar to the distribution of "ground glass" and orcein positive hepatocytes.

Ammunoperoxidase staining given by the antibodies anti-HBs (panel $19 / 10(D)$, anti-pre-S1 TO 606 (E). This pattern of decreasing intensity $(A-E)$ was seen in nine of 15 biopsy specimens and in the control wedge biopsy specimen. 
Discussion

This study shows that both pre-S1 and pre-S2 polypeptides can be detected in paraffin wax embedded liver tissue, confirming and expanding the findings of a previous study. ${ }^{17}$ It also shows that identification of pre-S antigen in paraffin wax sections largely depends on the reagents used.

Successful detection of pre-S polypeptides in paraffin wax embedded material has been previously reported using two of the monoclonal antibodies used in the present study, with similar results. ${ }^{17}$ Thus, while we detected pre-S1 in all but two biopsy specimens using MA 18/7, and pre-S2 in eight of our 15 specimens-weakly in five using $Q 19 / 10$, Dienes et al, using the same reagents, found pre-S1 in $75 \%$ of their cases and weak pre-S2 in a smaller, unspecified, proportion of specimens. ${ }^{17}$ When, however, we used TO 606 and 5535, which recognise different epitopes on pre-S1 and pre-S2, we found that pre-S2 was present in all the specimens and pre-S1 in only one of the 15 samples. Thus the intensity of staining and the number of positive cells clearly depend on the reagents used because antibodies of similar broad specificity give very different results, at least when used on paraffin wax embedded material. This difference may be explained by different affinities of the monoclonal antibodies used or by the fact that epitopes on intrahepatocytic pre-S antigens vary in their accessibility to antibodies or in their vulnerability to paraffin wax embedding. That pre-S epitopes behave differently when exposed to histological manoeuvres is illustrated by the finding that mild enzymatic digestion induced an appreciable decrease in the detectability of three of four determinants targeted by our panel of monoclonal antibodies.

The relation between the expression of pre-S polypeptides and HBV replicative activity has been investigated in previous studies with conflicting results. Theilman et al suggested that the presence of pre-S1 in the serum and liver is indicative of an intense HBV replication, ${ }^{18}$ while, according to Budkowska et al, ${ }^{19}$ it is serum pre-S2 that correlates with viral replication. In our study the expression of pre$\mathrm{S} 1$ and pre-S2 in the liver did not correlate with viral replication, because both polypeptides could be detected, irrespective of whether the patients had e antigen or e antibody in their sera, or core antigen in their livers. This lack of correlation agrees with the findings of Fraiese $e t$ $a l^{20}$ and Hadziyannis et $a l,{ }^{21}$ who were also unable to associate the tissue expression of pre$S$ polypeptides with $\mathrm{HBV}$ replicative activity.

Budkowska et al suggested that pre-S2 polypeptides could be a marker of disease activity because their presence in the liver was found in the most severe of the HBV-related liver disorders. ${ }^{19}$ In our series where diagnoses ranged from "healthy" carrier to chronic active hepatitis, we observed no correlation between the presence of pre-S1 and pre-S2 polypeptides and the degree of liver damage. As described for the surface antigen, however, an extensive positivity for either polypeptide tended to be a trait of the less severe conditions such as chronic persistent hepatitis and "healthy" chronic carriers. Larger studies are needed to substantiate this association and to clarify what role pre-S polypeptides have in the pathogenesis of HBV-related liver diseases.

In conclusion, the finding that pre-S1 and pre-S2 can be detected readily in paraffin wax embedded tissue opens the prospect for their characterisation, in stored material. The observation that reagents of similar broad specificities produced widely different results advocates the use of a panel of monoclonal antibodies in the study of pre-S polypeptides, at least on paraffin wax embedded material.

NH was supported by a British Council Research Fellowship. the photomicrographs.

1 Tiollais P, Pourcel C, Dejean A. The hepatitis B virus. Nature 1985;317:489-95.

2 Gerber MA, Thung SN. Molecular and cellular pathology of hepatitis B. Lab Invest 1985;52:572-90.

3 Neurath R, Kent S, Strick N, Parker K. Biological role pre-S sequences of the hepatitis $B$ virus envelope protein. In Robinson W, Koike K, Will H, eds. Hepadna viruses. New York: Alan R Liss Inc, 1987:189-203.

4 Gerlich HW, Bruss V, Heermann KH, Marquardt O, Seifer $M$. Presurface and precore products of human hepatitis B virus. In: Robinson W, Koike K, Will H, eds. hepadna viruses. New York: Alan R Liss Inc, 1987: Hepadna

5 Neurath AR, Kent SBH, Strick N, et al. Identification and chemical synthesis of a host cell receptor binding site on hepatitis B virus. Cell 1986;46:429-36.

6 Imai M, Yanase Y, Nojiri T, et al. A receptor for polymerized human and chimpanzee albumins on hepatitis B virus particles co-occurring with $\mathrm{HBeAg}$ Gastroenterology 1979;76:242-7.

7 Thung SN, Gerber MA. HBsAg-associated albumin receptors and anti-albumin antibodies in sera of patients with liver disease. Gastroenterology 1981;80:260-4

8 Gerber MA, Thung SN. The pre-S2 region of Hepatitis B Virus: more questions than answers. Hepatology 1989; 9:328-30.

9 Winckler K, Junge U, Creutzfeldt W. Ground-Glass hepatocytes in unselected liver biopsies. Ultrastructure and relationship to hepatitis B surface antigen. Scand and relationship to hepatitis B

10 Deodhar KP, Tapp E, Scheuer PJ. Orcein staining of hepatitis $\mathrm{B}$ antigen in paraffin sections of liver biopsies $J$ Clin Pathol 1975;28:66-70.

11 Portmann B, Galbraith RM, Eddleston ALW, Zuckerman AJ, Williams R. Detection of HBsAg in fixed liver tissue-use of a modified immunofluorescent technique and comparison with histochemical methods. Gut 1976;17:1-9.

12 Thomsen P, Poulsen H, Petersen P. Different types of ground glass Hepatocytes in human liver biopsies: Morphology, occurrence and diagnostic significance. Scand J Gastroenterol 1976;11:113-9.

13 Hadziyannis S, Gerber AM, Vissoulis C, Popper H. Cytoplasmic Hepatitis B antigen in "ground-glass" plasmic Hepatitis B antigen in "ground-glass"

14 Leevy CM, Popper H, Sherlock S. Diseases of the liver and biliary tract: standardisation of nomenclature, diagnostic criteria and diagnostic methodology. Sponsored by the John E Fogarty Centre for Advanced Study in Health Services and International Association for the Study of the Liver. Proceeding No 22. Washington DC: National Institute of Health, 1976:10 (DHEW publication No (NIH) 76-725)

15 Vergani D, Masera G, Moroni G, et al. Histological evidence of hepatitis-B-virus infection with negative serology in children with acute leukaemia who develop chronic liver disease. Lancet 1982;i:361-4.

16 Heermann $\mathrm{KH}$, Goldmann $U$, Schwartz $W$, et al. Large surface proteins of hepatitis B virus containing the pre-S surface proteins of hepatitis B virus
sequence. $J$ Virol 1984;52:396-402.

17 Dienes PH, Bianchi L, Gerlich W, Hess G. Different patterns of $\mathrm{HBsAg}$ and pre-S antigens in liver biopsies from healthy carriers. In: Zuckerman AJ, ed. Viral hepatitis and liver disease. London: Alan R Liss Inc, 1988:290-1

18 Theilman L, Klinkert M, Gmelin K, Salfeld J, Schaller H, Pfaff E. Detection of Pre-S1 proteins in serum and liver of HBsAg-positive patients: a new marker for hepatitis B virus infection. Hepatology 1986;6:186-90.

19 Budkowska A, Dubrenil P, Pillot J. Prognostic value of preS2 epitopes of hepatitis B virus and anti-pre-S2 response evaluated by monoclonal assays. In: Zuckerman AJ, ed. Viral hepatitis and liver disease. London: Alan R Liss Inc, 1988:287-9.

20 Fraiese A, Pontisso P, Cavalletto D, Fattowich G, Alberti A Expression of pre-S1 and pre-S2 in the liver of chronic hepatitis B virus carriers. J Hepatol 1988;7:157-63.

21 Hadziyannis S, Raimondo G, Papaioannou C, et al. Expression of pre-S gene-encoded proteins in liver and serum
during chronichepatitis B virus infection in comparison to during chronic hepatitis $\mathrm{B}$ virus infection in comparison to 1987;5:253-9. 